\title{
Linear Constrained Moving Horizon Estimator With Pre-Estimating Observer
}

\author{
D. Sui ${ }^{\mathrm{a}}$, T. A. Johansen ${ }^{\mathrm{b}}$ \\ ${ }^{a}$ Department of Petroleum Engineering, University of Stavanger, Norway (e-mail: \\ dan.sui@uis.no). \\ ${ }^{b}$ Center for Autonomous Marine Operations and Systems, Department of Engineering \\ Cybernetics, Norwegian University of Science and Technology, Trondheim, Norway.
}

\begin{abstract}
In this paper, a constrained moving horizon estimation (MHE) strategy for linear systems is proposed. Recently, the use of a pre-estimating linear observer in the forward prediction equations in the MHE cost function has been proposed in order to reduce the effects of uncertainty. Here we introduce state constraints within this formulation, and investigate stability properties in the presence of bounded disturbances and noise. The robustness and performance of the proposed observer is demonstrated with a simulation example.
\end{abstract}

Keywords: Moving Horizon Estimation; State Constraints; Input-to-state Stability.

\section{Introduction}

Moving horizon state estimator (MHE) has recently received much attention in the literature, e.g. Rao et al. (2001, 2003); Alessandri et al. (2003, 2004). The idea of MHE is to estimate the current states by solving a least squares optimization problem, which penalizes the deviation between the measurements and predicted outputs, and possibly the distance from the estimated state and an a priori state estimate. Since MHE is based on a window of the most recent data, it can provide a high degree of robustness in the presence of uncertainties such as noise, disturbances and modeling errors, see Alessandri et al. (2003).

Recently, the authors proposed an improved linear MHE approach (Sui et al., 2010) with a pre-estimating linear estimator instead of open loop forward prediction equations normally being used in the cost functions and constraints of the MHE. This development was made in spirit to the so-called 'pre-stabilizing' 
model predictive control (Rossiter et al., 1998; Kouvaritakis et al., 2000), where the control sequence is parameterized as perturbations to a given pre-stabilizing feedback gain. In the MHE, the injection term is parameterized as perturbations to a linear pre-estimating observer. Hence, the states are estimated by a forward simulation with a pre-estimator before optimized in MHE. Its motivation is to reduce the accumulation of estimation errors on the horizon by using output injection feedback for stabilization and shaping the dynamics. Moreover, an important effect of the pre-estimation approach compared to e.g. Rao et al. (2001) is that additional variables may not always need to be introduced in the optimization problem in order to account for the unknown disturbances that in our case are implicitly estimated and to some extent accounted for by the pre-estimating linear observer. This greatly reduces the computational complexity of the approach. The use of pre-estimation distinguishes the approach in Sui et al. (2010) and the present paper from the approaches known in the literature, e.g. Rao et al. (2001, 2003); Alessandri et al. (2003, 2004). The pre-estimator leads to a different set of tuning parameters that can be used to tune the performance, possibly achieving better performance than other approaches that are parameterized and tuned differently.

In this paper, the method of Sui et al. (2010) is extended to take into account state constraints, which can be expected to improve performance as in Rao et al. (2001, 2003); Haseltine and Rawlings (2005). Moreover, instead of using scalar weight parameters, weight matrices are introduced into the cost function. It is shown that the estimated error is input-to-state-stable (ISS) with respect to measurement noise and disturbance inputs.

The outline of the paper is as follows: After the introduction, some preliminaries are given in Section 2. The proposed constrained linear MHE is formulated in Section 3, and its convergence is investigated in Section 4. The robust performance of the proposed observer is demonstrated in a simulation example in Section 5, before some conclusions are given in Section 6.

\subsection{Notation and nomenclature}

A positive definite (semi-positive definite) square matrix $A$ is denoted by $A>0$ $(A \geq 0)$. $\|x\|_{A}^{2}=x^{T} A x$ with $A \geq 0$. Let $\rho(A)$ denote the spectral radius of a square matrix $A$. For two vectors $x \in R^{n}$ and $y \in R^{m}$ we let $\operatorname{col}(x, y)$ denote the column vector in $R^{n+m}$ where $x$ and $y$ are stacked into a single column. A set $X \subset R^{n}$ is said to be a $\mathscr{C}$ set if it is a compact and convex set that contains the origin in its (non-empty) interior. Suppose $X, Y \subset R^{n}$, then the $P$-difference of $X$ and $Y$ is 
$X \ominus Y=\left\{z \in R^{n}: z+y \in X, \forall y \in Y\right\}$. A function $\varphi: R^{+} \rightarrow R^{+}$is called a $K$ function if $\varphi(0)=0$ and it is strictly increasing. A function $\varphi: R^{+} \rightarrow R^{+}$is called a $K_{\infty}$-function if $\varphi \in K$ and it is radially unbounded. A function $\beta: R^{+} \times R^{+} \rightarrow R^{+}$ is called a $K L$-function if for each fixed $k \in R^{+}, \beta(\cdot, k) \in K$ and for each fixed $s \in R^{+}, \beta(s, \cdot)$ is non-increasing and $\lim _{k \rightarrow \infty} \beta(s, k)=0$. For a sequence $\left\{z_{j}\right\}$ for $j \geq 0, z_{[t]}$ denotes the truncation of $\left\{z_{j}\right\}$ at time $t$, i.e. $z_{[t]}=\left\{z_{j}\right\}$ for $0 \leq j \leq t$. A polyhedron is the (convex) intersection of a finite number of open and/or closed half-spaces and a polytope is the closed and bounded polyhedron.

\section{Background}

Consider the following discrete-time linear time-invariant system

$$
\begin{aligned}
x_{t+1} & =A x_{t}+B u_{t}+\xi_{t}, \\
y_{t} & =C x_{t}+\eta_{t},
\end{aligned}
$$

where $x_{t} \in X \subseteq R^{n_{x}}, u_{t} \in R^{n_{u}}$ and $y_{t} \in R^{n_{y}}$ are the state, input and the measurement, respectively, $\xi_{t} \in R^{n_{x}}$ is an unknown disturbance, $\eta_{t} \in R^{n_{y}}$ is unknown measurement noise, and $t$ is the discrete time index. It is assumed the disturbance $\xi_{t}$ and noise $\eta_{t}$ lie in the $\mathscr{C}$ sets $\Xi$ and $\Sigma$, respectively, and $X$ is a known set which will be used to define state constraints on the state estimation problem. We assume the input and measurement data are bounded.

While Sui et al. (2010) considers detectable systems, we consider without loss of generality observable systems:

(A1) the pair $(A, C)$ is observable.

By decomposing a detectable linear system into observable and unobservable subsystems, the present design approach is directly extended with an open loop observer for the asymptotically stable unobservable subsystem.

\subsection{Linear observer}

A linear time-invariant filter (e.g. Luenberger observer or stationary Kalman Filter) estimates the state according to

$$
\begin{aligned}
\hat{x}_{t+1} & =A \hat{x}_{t}+B u_{t}+L\left(y_{t}-\hat{y}_{t}\right), \\
\hat{y}_{t} & =C \hat{x}_{t},
\end{aligned}
$$

where $\hat{x}_{t} \in R^{n_{x}}$ is the current observer state, $\hat{y}_{t} \in R^{n_{y}}$ is the current observer output estimate, and the observer gain matrix is defined by $L \in R^{n_{x} \times n_{y}}$ such that the error dynamics characterized by $\Phi:=A-L C$ are asymptotically stable: 
(A2) $L$ satisfies $\rho(\Phi)<1$.

The estimated state $\hat{x}$ satisfies the following uncertain dynamics

$$
\hat{x}_{t+1}=A \hat{x}_{t}+B u_{t}+L C \tilde{e}_{t}+L \eta_{t},
$$

while the state estimation error $\tilde{e}_{t}=x_{t}-\hat{x}_{t}$ satisfies

$$
\tilde{e}_{t+1}=\Phi \tilde{e}_{t}+d_{t}
$$

where $d_{t}=\xi_{t}-L \eta_{t}$. Thus there always exists a $\mathscr{C}$ set $D$ such that $d_{t} \in D$.

Definition 1. (Kolmanovsky and Gilbert, 1998) A set $T \subset R^{n_{x}}$ is disturbance invariant (d-invariant) for the system $x_{t+1}=A x_{t}+\xi_{t}$ and the constraint set $(X, \Xi)$ if $T \subseteq X$ and $x_{t+1} \in T$ for all $\xi_{t} \in \Xi$ and $x_{t} \in T$.

Due to $\rho(\Phi)<1$, there exists a set $E$ such that it is $d$-invariant for the system (5). It implies that if $\tilde{e}_{0} \in E$, then $\tilde{e}_{t} \in E, \forall t \geq 0$. In this paper the set $E$ is chosen as the outer bound of the minimal $d$-invariant set of system (5). Methods to compute such a set for linear systems have appeared in the literature, see for example, Ong and Gilbert (2006); Raković et al. (2005).

\subsection{Input-to-state stability}

The concept of input-to-state stability (ISS) has been widely used in stability analysis and control synthesis. Recently, some of the well established results in ISS for continuous time nonlinear system have been extended to discrete-time nonlinear systems (Jiang and Wang, 2001). Some of the results of ISS are reviewed in this section.

Consider the following discrete-time nonlinear system:

$$
z_{t+1}=g\left(z_{t}, u_{t}\right)
$$

or $z^{+}=g(z, u)$, where $z_{t} \in Z \subseteq R^{n_{z}}$ and $u_{t} \in U$. We define the notion of input-tostate stability(Jiang and Wang, 2001).

Definition 2. The system (6) is ISS, if there exists a KL-function $\tilde{\theta}$, and a $K_{\infty}$ function $\gamma_{u}$ such that for any $t \geq 0$, any initial conditions $z_{0} \in X$ and any $u_{[t-1]}$ with $u_{j} \in U, 0 \leq j \leq t-1$, then the following is true:

$$
\left\|z_{t}\right\| \leq \tilde{\theta}\left(\left\|z_{0}\right\|, t\right)+\gamma_{u}\left(\left\|u_{[t-1]}\right\|\right)
$$


Definition 3. A continuous function $V: R^{n_{z}} \rightarrow R \geq 0$ is called an ISS-Lyapunov function for the system (6) if the following holds:

1. $V(0)=0$.

2. There exist $K_{\infty}$-functions $\alpha_{1}, \alpha_{2}$ such that for any $z$,

$$
\alpha_{1}(\|z\|) \leq V(z) \leq \alpha_{2}(\|z\|)
$$

3. There exists a $K$-function $\sigma$, such that for any $z$ and any input signals $u$

$$
V\left(z^{+}\right)-V(z) \leq-\alpha_{3}(\|z\|)+\sigma(\|u\|)
$$

or

$$
V\left(z^{+}\right)-V(z) \leq-\alpha_{3}\left(\left\|z^{+}\right\|\right)+\sigma(\|u\|)
$$

with $\alpha_{3}$ positive on $R^{+}$.

Theorem 1. (Jiang and Wang, 2001) If the system (6) admits an ISS-Lyapunov function, then it is ISS.

Note that an ISS system is globally asymptotically stable in absence of input or if the input is decaying. If the input is merely bounded then the evolution of the system is ultimately bounded in a set which size depends on the bound of the input.

\section{Linear Moving Horizon Estimation}

An MHE recursively estimates the state based on a finite window of current and past data. It estimates the state vectors $x_{t-N}, \ldots, x_{t}$ at any time $t=N, N+$ $1, \ldots$, on the basis of the a priori estimate $\bar{x}_{t-N, t}$ and the current information vector defined as

$$
I_{t}=\operatorname{col}\left(y_{t-N}, \ldots, y_{t}, u_{t-N}, \ldots, u_{t-1}\right),
$$

where $N+1$ is the window length or horizon.

Like in Sui et al. (2010), we formulate the MHE strategy by introducing a linear observer as a pre-estimating observer, since the injection term will reduce the effect of uncertainty (e.g. model errors, disturbances and noise) in the a priori estimate and predictions, and thereby contribute to improve the accuracy. The proposed MHE problem is formulated as follows to minimize with respect to $\hat{x}_{t-N, t}$

$$
J\left(\hat{x}_{t-N, t} ; \bar{x}_{t-N, t}, I_{t}\right)=\left\|y_{t-N}^{t}-\hat{y}_{t-N, t}^{t, t}\right\|_{W}^{2}+\left\|\hat{x}_{t-N, t}-\bar{x}_{t-N, t}\right\|_{M}^{2}
$$


subject to

$$
\begin{aligned}
\hat{x}_{i+1, t} & =A \hat{x}_{i, t}+B u_{i}+L\left(y_{i}-\hat{y}_{i, t}\right), \quad i=t-N, \ldots, t-1, \\
\hat{y}_{i, t} & =C \hat{x}_{i, t}, \quad i=t-N, \ldots, t, \\
\hat{x}_{i, t} & \in X, \quad i=t-N, \ldots, t
\end{aligned}
$$

where $W>0, M>0$ are weight matrices, $\rho(\Phi)<1, y_{t-N}^{t}=\operatorname{col}\left(y_{t-N}, \ldots, y_{t}\right)$ and $\hat{y}_{t-N, t}^{t, t}=\operatorname{col}\left(\hat{y}_{t-N, t}, \ldots, \hat{y}_{t, t}\right)$. The optimal solution of (11) is defined by $\hat{x}_{t-N, t}^{o}$ and it explicitly yields the sequence of the state estimates $\hat{x}_{i, t}^{o}, i=t-N+1, \ldots, t$ from (11b). It is assumed that the a priori estimator is determined from $\hat{x}_{t-N-1, t-1}^{o}$, that is

$$
\begin{aligned}
\bar{x}_{t-N, t} & =A \hat{x}_{t-N-1, t-1}^{o}+B u_{t-N-1}+L\left(y_{t-N-1}-\hat{y}_{t-N-1, t-1}^{o}\right), \\
\hat{y}_{t-N-1, t-1}^{o} & =C \hat{x}_{t-N-1, t-1}^{o} .
\end{aligned}
$$

Then $\bar{x}_{t-N, t}$ can be written as $\bar{x}_{t-N, t}=\Phi \hat{x}_{t-N-1, t-1}^{o}+B u_{t-N-1}+L y_{t-N-1}$.

In order to be able to implement the MHE using quadratic programming, the constraint set $X$ needs to be polyhedral, and feasibility is directly guaranteed by assuming the true state belongs to this set with some margin:

(A3) $X$ is a polyhedral set.

(A4) The state satisfies $x_{t} \in X \ominus E$ for all $t \geq 0$.

The state constraint set is reduced by the P-difference $X \ominus E$ in assumption (A4), in order to have sufficient margin to guarantee feasibility on the horizon despite disturbances and measurement noise. Clearly, the size of the set $E$ depends on the magnitude of disturbances and measurement noise. The choice of weight matrices $W$ and $M$ will be discussed in the next sections.

\section{Stability analysis}

The estimation error is defined as

$$
e_{t-N}=x_{t-N}-\hat{x}_{t-N, t}^{o}
$$


In order to state the stability results on the estimation error dynamics, we define

$$
\begin{aligned}
& F_{N}=\left[\begin{array}{c}
C \\
C \Phi \\
\vdots \\
C \Phi^{N}
\end{array}\right], \tilde{F}_{N}=\left[\begin{array}{c}
C \\
C A \\
\vdots \\
C A^{N}
\end{array}\right], G_{N}=\left[\begin{array}{llll}
0 & 0 & \cdots & 0 \\
C B & 0 & \cdots & 0 \\
C \Phi B & C B & \cdots & 0 \\
\vdots & \vdots & \ddots & \vdots \\
C \Phi^{N-1} B & C \Phi^{N-2} B & \cdots & C B
\end{array}\right] \\
& \tilde{G}_{N}=\left[\begin{array}{llll}
0 & 0 & \cdots & 0 \\
C B & 0 & \cdots & 0 \\
C A B & C B & \cdots & 0 \\
\vdots & \vdots & \ddots & \vdots \\
C A^{N-1} B & C A^{N-2} B & \cdots & C B
\end{array}\right] \\
& L_{N}=\left[\begin{array}{lllll}
0 & 0 & \cdots & 0 & 0 \\
C L & 0 & \cdots & 0 & 0 \\
C \Phi L & C L & \cdots & 0 & 0 \\
\vdots & \vdots & \ddots & \vdots & \vdots \\
C \Phi^{N-1} L & C \Phi^{N-2} L & \cdots & C L & 0
\end{array}\right], Q_{N}=I-L_{N} \\
& H_{N}=\left[\begin{array}{llll}
0 & 0 & \cdots & 0 \\
C & 0 & \cdots & 0 \\
\vdots & \vdots & \ddots & \vdots \\
C \Phi^{N-1} & C \Phi^{N-2} & \cdots & C
\end{array}\right], \tilde{H}_{N}=\left[\begin{array}{llll}
0 & 0 & \cdots & 0 \\
C & 0 & \cdots & 0 \\
\vdots & \vdots & \ddots & \vdots \\
C A^{N-1} & C A^{N-2} & \cdots & C
\end{array}\right] \text {, } \\
& \Delta_{1}=\left[0, H_{N}, 0, Q_{N}\right], \Delta_{2}=[I, 0,-L, 0], \mathbf{w}_{t-N}=\operatorname{col}\left(\xi_{t-N-1}, \ldots, \xi_{t-1}, \eta_{t-N-1}, \ldots, \eta_{t}\right) \text {. }
\end{aligned}
$$

First, we establish lower and upper bounds on the optimal cost function $J_{t}^{o}=$ $J\left(\hat{x}_{t-N, t}^{o} ; \bar{x}_{t-N, t}, I_{t}\right)$ :

\section{Lemma 1.}

$$
J_{t}^{o} \leq\left\|\boldsymbol{w}_{t-N}\right\|_{\Delta_{1}^{T} W \Delta_{1}}^{2}+\left\|\Phi e_{t-N-1}+\Delta_{2} \boldsymbol{w}_{t-N}\right\|_{M}^{2} .
$$

Proof. In the proof, we first show that $x_{t-N}$ is a feasible solution of MHE problem (11). According to the system (11b)-(11c) with the initial state $x_{t-N}$, the subsequential estimated states can be expressed by

$$
\begin{aligned}
\hat{x}_{i+1, t}^{f} & =A \hat{x}_{i, t}^{f}+B u_{i}+L\left(y_{i}-\hat{y}_{i, t}^{f}\right), \hat{x}_{t-N, t}^{f}=x_{t-N}, \quad i=t-N, \ldots, t-1, \\
\hat{y}_{i, t}^{f} & =C \hat{x}_{i, t}^{f}, \quad i=t-N, \ldots, t .
\end{aligned}
$$


Let $\tilde{e}_{i, t}=x_{i}-\hat{x}_{i, t}^{f}, \quad i=t-N, \ldots, t$. Since (A4) holds, we know that $x_{i} \in X \ominus E$ for $i=t-N, \ldots, t$. Consequently, $\hat{x}_{i, t}^{f}+\tilde{e}_{i, t} \in X \ominus E, \quad i=t-N, \ldots, t$. From the discussion in Section 2.1, we know the error $\tilde{e}_{i, t}$ satisfies the dynamics (5) and is within a d-invariant set $E$, or $\tilde{e}_{i, t} \in E$. Then we have $\hat{x}_{i, t}^{f} \in X, \forall \tilde{e}_{i, t} \in E$. Therefore, we know that $x_{t-N}$ is a feasible solution of the problem (11), and from the optimality of $\hat{x}_{t-N, t}^{o}$, we have $J_{t}^{o} \leq J\left(x_{t-N} ; \bar{x}_{t-N, t}, I_{t}\right)$. It follows that

$$
\begin{aligned}
& y_{t-N}^{t}=\tilde{F}_{N} x_{t-N}+\tilde{G}_{N} u_{t-N}^{t-1}+\tilde{H}_{N} \xi_{t-N}^{t-1}+\eta_{t-N}^{t}, \\
& \tilde{y}_{t-N}^{t}=F_{N} x_{t-N}+G_{N} u_{t-N}^{t-1}+L_{N} y_{t-N}^{t},
\end{aligned}
$$

where $\tilde{y}_{t-N}^{t}=\operatorname{col}\left(\hat{y}_{t-N, t}^{f}, \ldots, \hat{y}_{t, t}^{f}\right), u_{t-N}^{t-1}=\operatorname{col}\left(u_{t-N}, \ldots, u_{t-1}\right), \xi_{t-N}^{t-1}=\operatorname{col}\left(\xi_{t-N}, \ldots\right.$, $\left.\xi_{t-1}\right)$ and $\eta_{t-N}^{t}=\operatorname{col}\left(\eta_{t-N}, \ldots, \eta_{t}\right)$. Then we have

$$
\begin{aligned}
Q_{N} y_{t-N}^{t} & =Q_{N} \tilde{F}_{N} x_{t-N}+Q_{N} \tilde{G}_{N} u_{t-N}^{t-1}+Q_{N} \tilde{H}_{N} \xi_{t-N}^{t-1}+Q_{N} \eta_{t-N}^{t} \\
& =F_{N} x_{t-N}+G_{N} u_{t-N}^{t-1}+H_{N} \xi_{t-N}^{t-1}+Q_{N} \eta_{t-N}^{t} .
\end{aligned}
$$

From the above, it is obtained that

$$
y_{t-N}^{t}-\tilde{y}_{t-N}^{t}=H_{N} \xi_{t-N}^{t-1}+Q_{N} \eta_{t-N}^{t}=\Delta_{1} \mathbf{w}_{t-N}
$$

and

$$
\left\|y_{t-N}^{t}-\tilde{y}_{t-N, t}^{t}\right\|_{W}^{2}=\left\|\mathbf{w}_{t-N}\right\|_{\Delta_{1}^{T} W \Delta_{1}}^{2} .
$$

Furthermore, since $x_{t-N}=A x_{t-N-1}+B u_{t-N-1}+\xi_{t-N-1}$ and $\bar{x}_{t-N, t}=\Phi \hat{x}_{t-N-1, t-1}^{o}+$ $B u_{t-N-1}+L y_{t-N-1}$, then

$$
x_{t-N}-\bar{x}_{t-N, t}=\Phi\left(x_{t-N-1}-\hat{x}_{t-N-1, t-1}^{o}\right)+\xi_{t-N-1}-L \eta_{t-N-1} .
$$

Therefore, we have

$$
\left\|x_{t-N}-\bar{x}_{t-N, t}\right\|_{M}^{2}=\left\|\Phi e_{t-N-1}+\Delta_{2} \mathbf{w}_{t-N}\right\|_{M}^{2} .
$$

Combining (19) and (20), we get (14).

\section{Lemma 2.}

$$
J_{t}^{o} \geq\left\|F_{N} e_{t-N}+\Delta_{1} \boldsymbol{w}_{t-N}\right\|_{W}^{2} .
$$


Proof. Taking zero as the lower bound on the second term of (11a) we get

$$
J_{t}^{o} \geq\left\|y_{t-N}^{t}-\hat{y}_{t-N, t}^{t, t}\right\|_{W}^{2}=\left(y_{t-N}^{t}-\hat{y}_{t-N, t}^{t, t}\right)^{T} W\left(y_{t-N}^{t}-\hat{y}_{t-N, t}^{t, t}\right) .
$$

Since

$$
y_{t-N}^{t}-\hat{y}_{t-N, t}^{t, t}=F_{N}\left(x_{t-N}-\hat{x}_{t-N, t}^{o}\right)+H_{N} \xi_{t-N}^{t-1}+Q_{N} \eta_{t-N}^{t}=F_{N} e_{t-N}+\Delta_{1} \mathbf{w}_{t-N},
$$

we have (21).

Theorem 2. There always exist matrices $W>0$ and $M>0$ such that the error $e_{t}$ is ISS. Moreover, when $\xi_{t}=0$ and $\eta_{t}=0, \forall t \geq 0$, the error dynamics $e_{t}$ is exponentially stable for any initial condition $\bar{x}_{-N, 0}$.

Proof. Since $\rho(\Phi)<1$, there always exists a matrix $M=M^{T}>0$ such that

$$
\Phi^{T} M \Phi-M \leq-Q_{1},
$$

for any $Q_{1}=Q_{1}^{T}>0$. Consider a Lyapunov function candidate

$$
V\left(e_{t-N}\right)=\left\|e_{t-N}\right\|_{M}^{2}
$$

In the following $V\left(e_{t-N}\right)-V\left(e_{t-N-1}\right)<0, \forall e_{t-N-1} \neq 0$ for some $W$ is shown. From (22), it is easy to show that

$$
\left\|e_{t-N-1}\right\|_{M}^{2} \geq\left\|\Phi e_{t-N-1}\right\|_{M}^{2}+\left\|e_{t-N-1}\right\|_{Q_{1}}^{2} .
$$

The first item of the right side of the above inequality can be expressed as

$$
\begin{aligned}
& \left\|\Phi e_{t-N-1}\right\|_{M}^{2} \\
& =\left\|\Phi e_{t-N-1}+\Delta_{2} \mathbf{w}_{t-N}-\Delta_{2} \mathbf{w}_{t-N}\right\|_{M}^{2} \\
& =\left\|\Phi e_{t-N-1}+\Delta_{2} \mathbf{w}_{t-N}\right\|_{M}^{2}+\left\|\Delta_{2} \mathbf{w}_{t-N}\right\|_{M}^{2} \\
& -\left(\Phi e_{t-N-1}+\Delta_{2} \mathbf{w}_{t-N}\right)^{T} M \Delta_{2} \mathbf{w}_{t-N}-\mathbf{w}_{t-N}^{T} \Delta_{2}^{T} M\left(\Phi e_{t-N-1}+\Delta_{2} \mathbf{w}_{t-N}\right) .
\end{aligned}
$$

Since the item $\left(\Phi e_{t-N-1}+\Delta_{2} \mathbf{w}_{t-N}\right)^{T} M \Delta_{2} \mathbf{w}_{t-N}$ is scaler and $M=M^{T}>0$, we have

$$
\begin{aligned}
& \left\|\Phi e_{t-N-1}\right\|_{M}^{2} \\
& =\left\|\Phi e_{t-N-1}+\Delta_{2} \mathbf{w}_{t-N}\right\|_{M}^{2}+\left\|\Delta_{2} \mathbf{w}_{t-N}\right\|_{M}^{2}-2\left(\Phi e_{t-N-1}+\Delta_{2} \mathbf{w}_{t-N}\right)^{T} M \Delta_{2} \mathbf{w}_{t-N} \\
& =\left\|\Phi e_{t-N-1}+\Delta_{2} \mathbf{w}_{t-N}\right\|_{M}^{2}-2 e_{t-N-1}^{T} \Phi^{T} M \Delta_{2} \mathbf{w}_{t-N}-\left\|\mathbf{w}_{t-N}\right\|_{\Delta_{2}^{T} M \Delta_{2}}^{2} .
\end{aligned}
$$


Therefore,

$$
\begin{aligned}
\left\|e_{t-N-1}\right\|_{M}^{2} \geq & \left\|\Phi e_{t-N-1}+\Delta_{2} \mathbf{w}_{t-N}\right\|_{M}^{2}-2 e_{t-N-1}^{T} \Phi^{T} M \Delta_{2} \mathbf{w}_{t-N}-\left\|\mathbf{w}_{t-N}\right\|_{\Delta_{2}^{T} M \Delta_{2}}^{2} \\
& +\left\|e_{t-N-1}\right\|_{Q_{1}}^{2} \\
\Rightarrow\left\|e_{t-N-1}\right\|_{M}^{2} \geq & \left\|\Phi e_{t-N-1}+\Delta_{2} \mathbf{w}_{t-N}\right\|_{M}^{2}+\delta_{1}\left(\left\|e_{t-N-1}\right\|\right)-\rho_{1}\left(\left\|\mathbf{w}_{t-N}\right\|\right) \\
\Rightarrow\left\|e_{t-N-1}\right\|_{M}^{2} \geq & \left\|\Phi e_{t-N-1}+\Delta_{2} \mathbf{w}_{t-N}\right\|_{M}^{2}-\rho_{1}\left(\left\|\mathbf{w}_{t-N}\right\|\right)
\end{aligned}
$$

where $\delta_{1}(r)=1 / 2 \lambda_{\min }\left(Q_{1}\right) r^{2}$ and $\rho_{1}(r)=\theta_{1} r^{2}$ with $\theta_{1}=\frac{2\left\|\Phi^{T} M \Delta_{2}\right\|^{2}}{\lambda_{\min }\left(Q_{1}\right)}+\left\|\Delta_{2}^{T} M \Delta_{2}\right\|^{2}$. Furthermore, combining (14) and (21), we get that

$$
\begin{aligned}
& \left\|\Phi e_{t-N-1}+\Delta_{2} \mathbf{w}_{t-N}\right\|_{M}^{2}+\left\|\mathbf{w}_{t-N}\right\|_{\Delta_{1}^{T} W \Delta_{1}}^{2} \geq\left\|F_{N} e_{t-N}+\Delta_{1} \mathbf{w}_{t-N}\right\|_{W}^{2} \\
\Rightarrow & \left\|\Phi e_{t-N-1}+\Delta_{2} \mathbf{w}_{t-N}\right\|_{M}^{2} \geq\left\|F_{N} e_{t-N}+\Delta_{1} \mathbf{w}_{t-N}\right\|_{W}^{2}-\left\|\mathbf{w}_{t-N}\right\|_{\Delta_{1}^{T} W \Delta_{1}}^{2} \\
\Rightarrow & \left\|\Phi e_{t-N-1}+\Delta_{2} \mathbf{w}_{t-N}\right\|_{M}^{2} \geq\left\|e_{t-N}\right\|_{F_{N}^{T} W F_{N}}^{2}+2 e_{t-N}^{T} F_{N}^{T} W \Delta_{1} \mathbf{w}_{t-N} .
\end{aligned}
$$

From (24) and (25), we have

$$
\left\|e_{t-N-1}\right\|_{M}^{2} \geq\left\|e_{t-N}\right\|_{F_{N}^{T} W F_{N}}^{2}+2 e_{t-N}^{T} F_{N}^{T} W \Delta_{1} \mathbf{w}_{t-N}-\rho_{1}\left(\left\|\mathbf{w}_{t-N}\right\|\right)
$$

From the above, we have

$$
\begin{aligned}
& V\left(e_{t-N}\right)-V\left(e_{t-N-1}\right)=\left\|e_{t-N}\right\|_{M}^{2}-\left\|e_{t-N-1}\right\|_{M}^{2} \\
& \leq\left\|e_{t-N}\right\|_{M}^{2}-\left\|e_{t-N}\right\|_{F_{N}^{T} W F_{N}}^{2}-2 e_{t-N}^{T} F_{N}^{T} W \Delta_{1} \mathbf{w}_{t-N}+\rho_{1}\left(\left\|\mathbf{w}_{t-N}\right\|\right) .
\end{aligned}
$$

Since $F_{N}$ has full rank due to (A1), there always exists a large enough matrix $W>0$ such that

$$
M-F_{N}^{T} W F_{N} \leq-Q_{2},
$$

for some $Q_{2}>0$. Therefore, we have

$$
\begin{aligned}
V\left(e_{t-N}\right)-V\left(e_{t-N-1}\right) & \leq-\left\|e_{t-N}\right\|_{Q_{2}}^{2}-2 e_{t-N}^{T} F_{N}^{T} W \Delta_{1} \mathbf{w}_{t-N}+\rho_{1}\left(\left\|\mathbf{w}_{t-N}\right\|\right) \\
& \leq-\delta_{2}\left(\left\|e_{t-N}\right\|\right)+\rho_{2}\left(\left\|\mathbf{w}_{t-N}\right\|\right)
\end{aligned}
$$

where $\delta_{2}(r)=1 / 2 \lambda_{\min }\left(Q_{2}\right) r^{2}$ and $\rho_{2}(r)=\left(\theta_{1}+\theta_{2}\right) r^{2}$ with $\theta_{2}=\frac{2\left\|F_{N}^{T} W \Delta_{1}\right\|^{2}}{\lambda_{\min }\left(Q_{2}\right)}$. Therefore, $e_{t}$ is ISS. Moreover, it is easy to show that when $\xi_{t}=0$ and $\eta_{t}=0, t=$ $0,1, \ldots$, the error dynamics $e_{t}$ is globally exponentially stable. 
Corollary 1. Suppose that assumptions (A1)-(A3) hold. If the weight matrices $M, W$ satisfy

$$
\begin{aligned}
\Phi^{T} M \Phi-M & \leq-Q_{1}, \\
M-F_{N}^{T} W F_{N} & \leq-Q_{2}, \\
M=M^{T} & >0, \\
W & >0,
\end{aligned}
$$

for some $Q_{1}>0, Q_{2}>0$, then the error dynamics $e_{t}$ is ISS.

From Corollary 1, there exists much flexibility to choose the weight matrices $W$ and $M$ in order to optimize performance. In the following, one way to determine $W$ and $M$ is given. Suppose the choice of $M=M^{T}>0$ satisfies the inequality (27a) for some $Q_{1}>0$.

Assuming all variables are reasonably scaled, we propose to choose the matrix $W=W_{1}^{T} W_{1}$ where $W_{1}$ is chosen to satisfy

$$
W_{1} F_{N}=\sqrt{\mu} I_{n_{x}}
$$

and $\mu>0$ is a scalar tuning parameter. Since the system is observable, this leads to the choice

$$
W_{1}=\sqrt{\mu} F_{N}^{+},
$$

where $F_{N}^{+}=F_{N}^{T}\left(F_{N}^{T} F_{N}\right)^{-1}$ is the pseudo-inverse. According to (27a), $\mu$ should be chosen such that $\mu I_{n_{x}}>M$. This tuning procedure is similar to the unconstrained case (Sui et al., 2010) as it is often not necessary to explicitly account for the constraints in the tuning.

\section{Example}

The example is taken from Haseltine and Rawlings (2005). The linearized discrete-time model of a well-mixed, constant volume, isothermal batch reactor is given by the system matrices, with sampling interval $t_{f}=0.25$ :

$$
A=\left[\begin{array}{lll}
0.8831 & 0.0078 & 0.0022 \\
0.1150 & 0.9563 & 0.0028 \\
0.1178 & 0.0102 & 0.9954
\end{array}\right], B=\left[\begin{array}{l}
0 \\
0 \\
0
\end{array}\right], C=[32.84,32.84,32.84]
$$


The sequentially independent disturbance $\xi_{t}$ is a $N(0, \mathscr{Q})$ noise with $\mathscr{Q}=$ $\operatorname{diag}\left(0.001^{2}, 0.001^{2}, 0.001^{2}\right)$ and $\eta_{t}$ is a $N(0, \mathscr{R})$ sequentially independent noise with $\mathscr{R}=0.25^{2}$, and $N(m, \Sigma)$ denotes a multi-variate normal distribution with mean $m$ and covariance matrix $\Sigma$. It is assumed that the state constraint is defined as $X=\{x \mid x \geq 0\}$, and the observer gain matrix $L=\left[\begin{array}{ll}0.0030 .009 & 0.00043\end{array}\right]^{T}$ is chosen such that $\rho(\Phi)<1$ and its eigenvalues are $0.4754,0.8497,0.9727$. Its window size has been chosen as $N=11 . M=0.25 I_{3}$ is chosen such that (27a) holds, and $\mu=0.5$. Let us consider the performance indexes given by the root mean square error (RMSE):

$$
R M S E=\left(\sum_{t=t_{P}}^{t_{S}} \frac{\left\|e_{t}\right\|^{2}}{t_{S}-t_{P}}\right)^{1 / 2},
$$

where $t_{S}$ is the length of each simulation run. We choose $t_{S}=120$ and $t_{P}=N$.

We consider a simulation scenario from Haseltine and Rawlings (2005) where the true initial state is $x_{0}=[0.5,0.05,0]^{T}$, and the initial guess is $\bar{x}_{0}=[0,0,4]^{T}$. The estimated $x$ and $y$ by the proposed MHE are shown in Figures 1 and 2 , Its RMSE is 0.0292 .

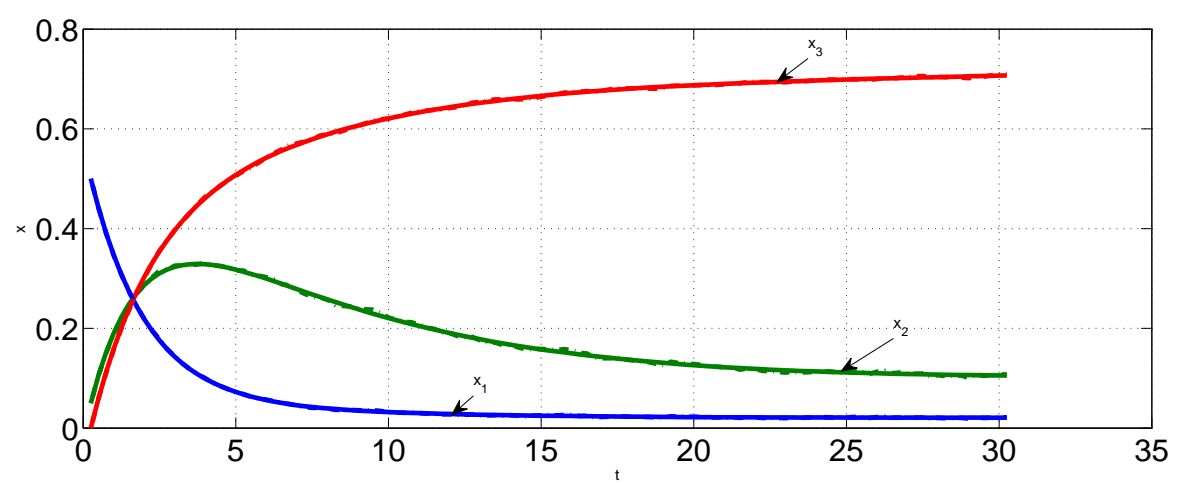

Figure 1: The evolution of the actual state(solid line) from the linearized model and proposed MHE estimation (dash-dot line).

Figures 3 , 4 show the estimated states and output based on the linearized system by using the clipped Kalman filter with the same initial state, initial guess and the same initial covariance matrix, where the negative filtered values of the state are set to zero (if $\hat{x}_{t, t}<0$, set $\hat{x}_{t, t}=0$ ). From the figures, it is seen that the estimated states converges to the true state, but the estimation during the first step is poor. Its RMSE is 0.384 . 


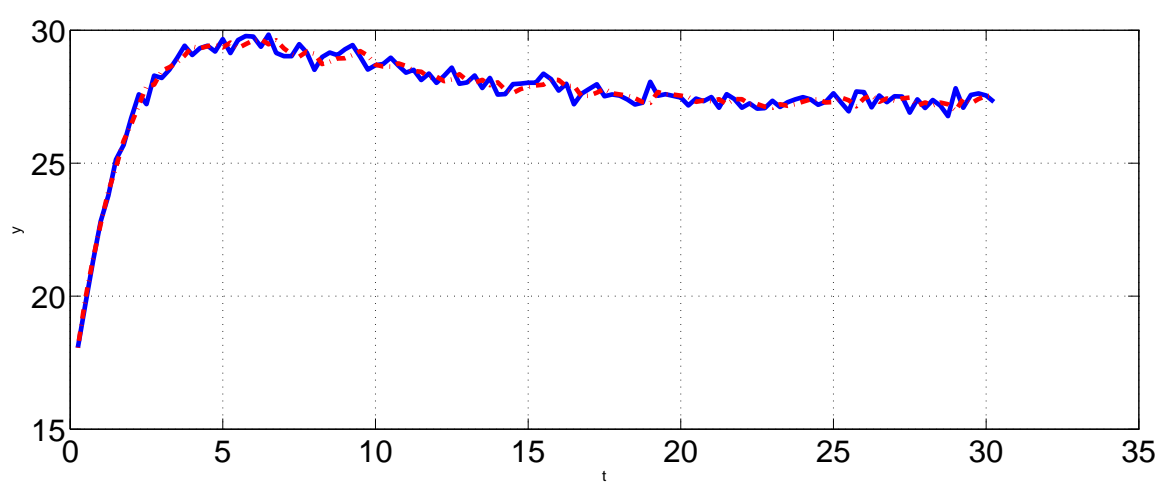

Figure 2: The evolution of the actual output(solid line) from the linearized model and proposed MHE estimation (dash-dot line).

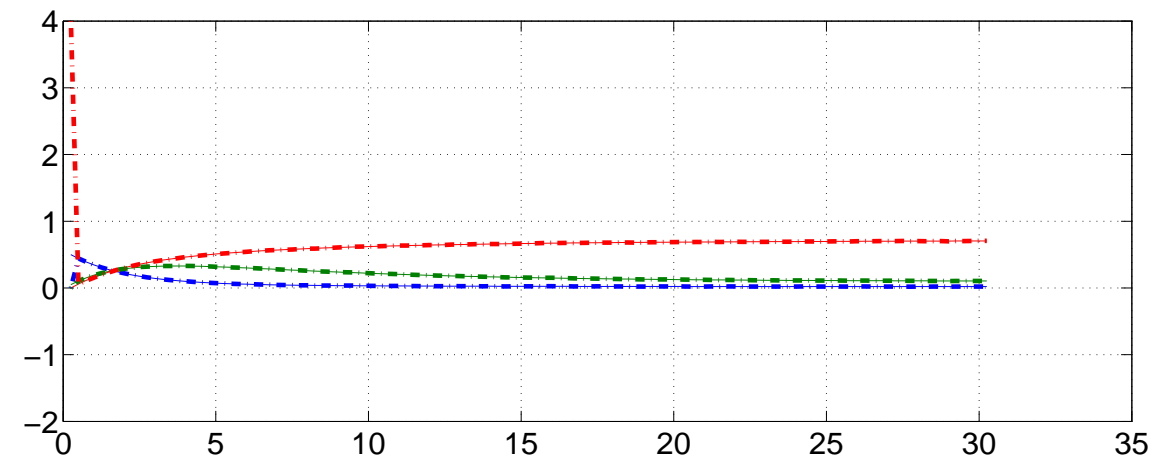

Figure 3: The evolution of the actual state(solid line) from the linearized model and clipped Kalman Filter(dash-dot line). 


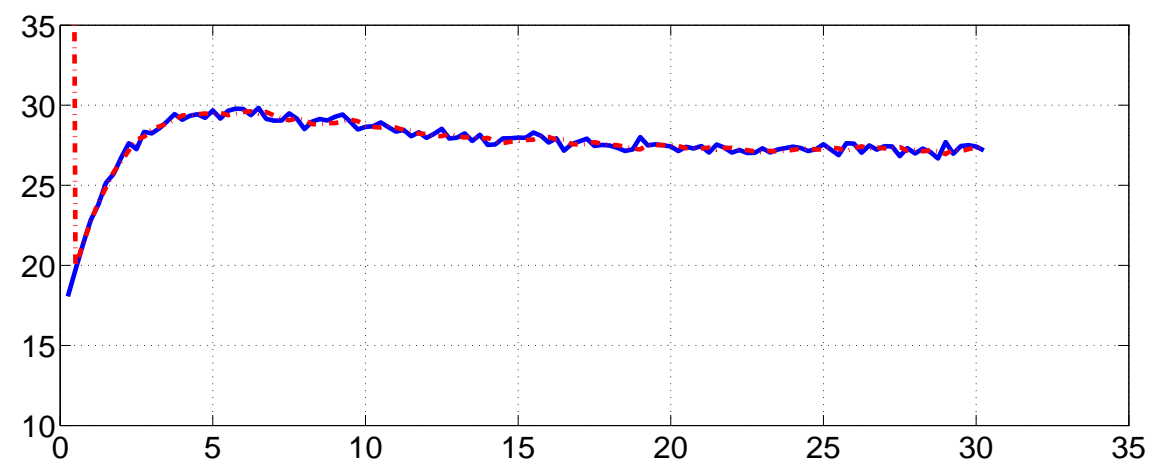

Figure 4: The evolution of the actual output(solid line) from the linearized model and clipped Kalman Filter(dash-dot line).

In Rao et al. (2001) the use of Kalman filter approximation of the arrival-cost in MHE is proposed since it is not generally possible to derive analytical expressions for the conditional density of constrained systems. Figures 5,6 plot such MHE approximation by using the same initial state and covariance matrix estimate, and the same horizon as above. Its RMSE is 0.0959. The performances of both two MHE approaches depend on, for instance, the choices of the weight matrices and the initial state covariance matrix of MHE in Rao et al. (2001). Therefore, it makes the direct comparisons of two MHE approaches not entirely straightforward.

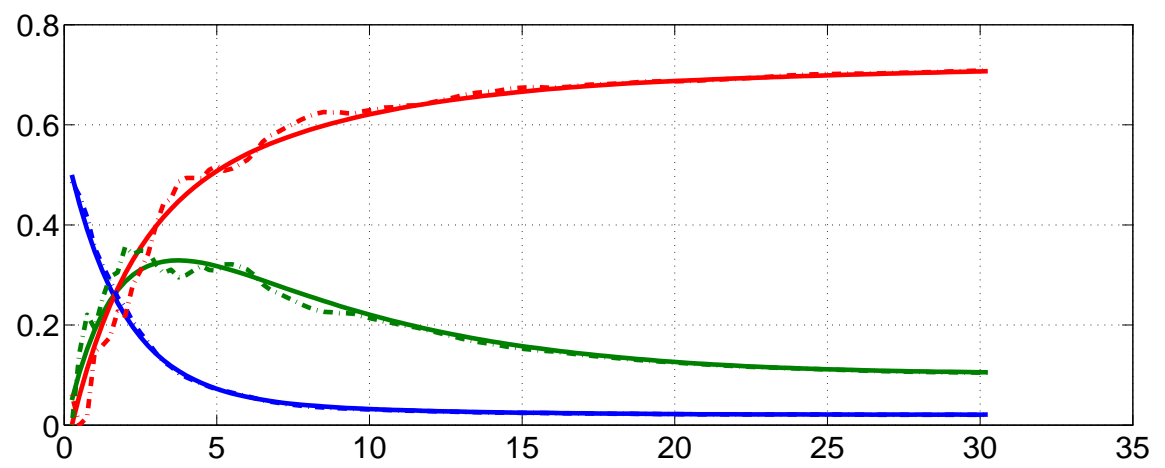

Figure 5: The evolution of the actual state(solid line) from the linearized model and MHE approximation via Rao et al. (2001)(dash-dot line). 


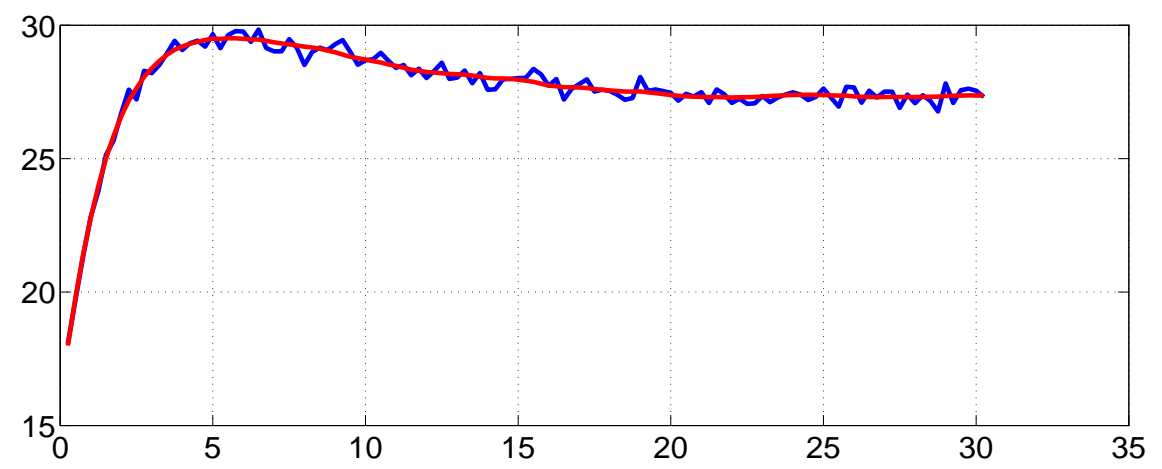

Figure 6: The evolution of the actual output(solid line)from the linearized model and MHE approximation via Rao et al. (2001) (dash-dot line).

\section{Conclusion}

In this paper, an MHE for linear systems with state constraints is proposed. Its unique feature is that it uses a pre-estimating linear filter within the forward prediction equations used in the MHE formulation. Hence, the MHE provides additional corrections to a standard linear injection term. Quite natural conditions for convergence and feasibility are derived, and the robustness of the method is studied in a simulation example where linear observer designs based on a linearized model of a chemical reactor are compared. It should be had in mind that beyond the good performance in the given example, a major benefit of the proposed approach is that the number of variables in the MHE optimization problem is fixed which is the dimension of the states, or, $n_{x}$. On the other hand, the number of variables of MHE approaches such as Rao et al. (2001) is determined by the dimension of the states, the dimension of the disturbance and the length of horizon, or, $(N+1) n_{x}$. Hence, the proposed method has a computational complexity that scales better with the horizon $N$.

\section{Acknowledgements}

This work is supported by the Center for Integrated Operations in the Petroleum Industry, the Research Council of Norway Strategic University Program on Computational Method in Nonlinear Motion Control, and the Research Council of Norway and Statoil through the Center of Excellence on Autonomous Marine Operations and Systems. 


\section{References}

Alessandri, A., Baglietto, M., Battistelli, G., 2003. Receding horizon estimation for discrete time linear systems. IEEE Transaction on Automatic Control 48.

Alessandri, A., Baglietto, M., Battistelli, G., 2004. On estimation error bounds for receding horizon filters using quadratic boundedness. IEEE Transactions on Automatic Control 49, 1350 - 1355.

Haseltine, E. L., Rawlings, J. B., 2005. Critical evaluation of extended kalman filtering and moving-horizon estimation. Industrial and Engineering Chemistry Research 44, 24512460.

Jiang, Z. P., Wang, Y., 2001. Input-to-state stability for discrete-time nonlinear systems. Automatica 37, 857-869.

Kolmanovsky, I., Gilbert, E., 1998. Theory and computation of disturbance invariance sets for discrecte-time linear systems. Mathematical Probems in Engineering: Theory, Methods and Applications 4, 317-367.

Kouvaritakis, B., Rossiter, J. A., Schuurmans, J., 2000. Efficient robust predictive control. IEEE Transactions on Automatic Control 45(8), 1545-1549.

Ong, C. J., Gilbert, E. G., 2006. The minimal disturbance invariant set: outer approximations via its partial sums. Automatica 42, 1563-1568.

Raković, S. V., Kerrigan, E. C., Kouramas, K. I., Mayne, D. Q., 2005. Invariant approximations of the minimal robust positively invariant set. IEEE Transactions on Automatic Control 50, 406-410.

Rao, C. V., Rawlings, J. B., Lee, J. H., 2001. Constrained linear state estimation: a moving horizon approach. Automatica 37, 1619-1628.

Rao, C. V., Rawlings, J. B., Mayne, D. Q., 2003. Constrained state estimation for nonlinear discrete-time systems: Stability and moving horizon approximation. IEEE Transactions Automatic Control 48, 246-258.

Rossiter, J. A., Kouvaritakis, B., Rice, M. J., 1998. A numerically robust statespace approach to stable predictive control strategies. Automatica 34, 65-73.

Sui, D., Johansen, T. A., Feng, L., 2010. Linear moving horizon estimation with pre-estimating observer. IEEE Transactions on Automatic Control 55, 23632368. 